\title{
THE FAMILY RUBIACEAE IN SOUTHERN ASSAM WITH SPECIAL REFERENCE TO ENDEMIC AND REDISCOVERED PLANT TAXA
}

\author{
H.A. Barbhuiya ${ }^{1}$, B.K. Dutta ${ }^{2}$, A.K. Das $^{3}$ \& A.K. Baishya ${ }^{4}$
}

ISSN

Online 0974-7907

Print 0974-7893

\author{
${ }^{1}$ Botanical Survey of India, Eastern Regional Centre, Shillong, Meghalaya 793003, India \\ 2,3 Department of Ecology and Environmental Science, Assam University, Silchar, Assam 788011, India \\ ${ }^{4}$ Laban, East Khasi Hills, Shillong, Meghalaya 793009, India \\ ${ }^{1}$ hussainbsi@yahoo.com (corresponding author), ${ }^{2}$ bimankdutta@rediffmail.com, ${ }^{3}$ ajitkumardas2009@rediffmail.com, \\ ${ }^{4}$ akbaishya@rediffmail.com
}

OPEN ACCESS

\begin{abstract}
Analysis of diversity, distribution and endemism of the family Rubiaceae for southern Assam has been made. The analyses are based on field observations in the three districts, viz., Cachar, Hailakandi and Karimganj, as well as data from existing collections and literature. The present study records 90 taxa recorded from southern Assam, four of which are endemic. Chassalia curviflora (Wall.) Thwaites var. ellipsoides Hook. f. and Mussaenda keenanii Hook.f. are rediscovered after a gap of 140 years. Mussaenda corymbosa Roxb. is reported for the first time from northeastern India, while Chassalia staintonii (H.Hara) Deb \& Mondal is reported as a new record for Assam.
\end{abstract}

Keywords: Assam, endemic, IUCN, new record, rediscovery, regional assessment, Rubiaceae.

The family Rubiaceae is widespread and occurs in all major regions of the world except Antarctica and shows maximum proliferation in low to mid altitude humid forests. The family comprises 13,143 species falling under 611 genera by which it is the fourth largest Angiosperm family (Davis et al. 2009). In India, it is represented by 551 species of which $67 \%$ taxa (365 spp.) are endemic (Sambamurthy 2005). Kar \& Panigrahi (1963) reported 118 species from Assam and the North East Frontier Agency (present Arunachal Pradesh), whilst Chowdhury (2005) listed ca. 150 species of Rubiaceae from the state of Assam.
Southern Assam (Barak Valley) is located between $24^{\circ} 08^{\prime}-25^{\circ} 08^{\prime} \mathrm{N}$ and $92^{\circ} 12^{\prime}-93^{\circ} 15^{\prime} \mathrm{E}$. The valley covers an area of $6,922 \mathrm{~km}^{2}$ and is surrounded by Dima Hasao District and Jaintia Hill in the north, the Manipur Hills in the east and the Mizoram Hills in the south. To the west the plains merge with the Sylhet plains of Bangladesh and the Indian state of Tripura. At present the region is divided into three administrative districts Cachar, Karimganj and Hailakandi. The $2,339 \mathrm{~km}^{2}$ forest area comprises of 12 reserve forests and one wildlife sanctuary, i.e., ca. $34 \%$ of the total geographical area of the valley. The drainage system of southern Assam is fed by 18 major rivers and rivulets and Barak is the chief river which flows in an eastwest direction through the central part of the valley. The annual average rainfall and temperature ranges from 2500-4000 mm and $7.5-40{ }^{\circ} \mathrm{C}$, respectively; whereas, the relative humidity varies from $60-91 \%$.

The vegetation of the region predominantly consists of tropical wet evergreen to tropical semi-evergreen forest types (Champion \& Seth 1968). Tropical wet evergreen type of forest is prevalent in the northern and eastern slopes of southern Assam like Borail Hill Ranges, Lower Jiri Reserve Forest, Barak RF, and Inner Line RF. The dominant tree species of this type of forest are Actinodaphne

DOI: http://dx.doi.org/10.11609/JoTT.o3117.5649-59

Editor: V. Sampath Kumar, Royal Botanic Gardens, Kew, UK

Date of publication: 26 April 2014 (online \& print)

Manuscript details: Ms \# 03117 | Received 09 March 2012 | Final received 08 March 2014 | Finally accepted 11 March 2014

Citation: H.A. Barbhuiya, B.K. Dutta, A.K. Das \& A.K. Baishya (2014). The family Rubiaceae in southern Assam with special reference to endemic and rediscovered plant taxa. Journal of Threatened Taxa 6(4): 5649-5659; http://dx.doi.org/10.11609/JoTT.03117.5649-59

Copyright: (c) Barbhuiya et al. 2014. Creative Commons Attribution 4.0 International License. JoTT allows unrestricted use of this article in any medium, reproduction and distribution by providing adequate credit to the authors and the source of publication.

Funding: Self funded.

Competing Interest: The authors declare no competing interests.

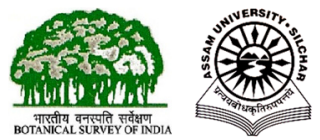

Acknowledgements: The first author is grateful to P. Singh, Director, Botanical Survey of India (BSI), Kolkata and to A.A. Mao, Scientist-E and Head of Office, BSI, Eastern Regional Centre, Shillong for facilities and encouragement. The authors also extends their sincere thanks to Board of Trustees of the Royal Botanic Gardens, Kew for providing the digital images of type specimens. 
obovata, Amoora rohituka, Dipterocarpus turbinatus, Duabanga grandiflora, Toona ciliata, Dysoxylum alatum, Melia azedarach, Mallotus nudiflorus, Mesua ferrea, Sterculia villosa, Quercus semiserrata, Callicarpa arborea, Castanopsis lanceifolia, Holarrhena antidysenterica, Schima wallichii, Macaranga indica, Bauhinia purpurea, Xerospermum glabratum, Mallotus albus and Ficus roxburghii.

Whereas tropical semi-evergreen type of forest is prevalent in the southern and western parts of southern Assam in Sonai RF, Katakhal RF, Singla RF, Badsha Tilla RF, Longai RF, Tilbum RF, Duhalia RF and Patheria RF. The dominant tree species of this type of forest are Schima wallichii, Saurauia roxburghii, Dipterocarpus turbinatus, Albizia procera, Gmelina arborea, Lagerstroemia speciosa, Macaranga denticulata, Mallotus nepalensis, $M$. roxburghianus, Phyllanthus emblica and Ficus bengalensis.

\section{Materials and Methods}

Field surveys were conducted from 2010 to 2013 in $2,339 \mathrm{~km}^{2}$ forest area, to assess the diversity, distribution, endemism and threats to the family Rubiaceae in different parts of southern Assam, particularly in reserve forests and wildlife sanctuaries. This was supplemented with a literature study of peer-reviewed papers, monographs and floras, followed by a study of the herbarium specimens housed at Botanical Survey of India, Eastern Regional Centre, Shillong (ASSAM). Digital images of type specimens were obtained from the Royal Botanical Gardens, Kew (UK) to confirm the identity of the species. Species distributions were plotted using Google Earth v.6.1.0.5001 (www.earth.google.com). Extent of Occurrence (EOO) were calculated by drawing a minimum convex polygon and areas were worked out by using the software GE Path v. 1.4.6 (Sgrillo 2012). Similarly Area of Occupancy (AOO) was calculated by the sum of the occupied isometric grid squares of size $20 \mathrm{~km}^{2}$ (Image 1B).

The regional taxa of southern Assam were then subjected to two step assessment procedure: In step one, criteria B \& D (IUCN 2001), were applied to the selected regional population of the selected taxon, resulting in a preliminary categorization. In step two, the existence and status of any conspecific populations outside the region that may affect the risk of extinction within the region was investigated either through field visit or based on information from herbarium data and published regional floras. If the taxon was found endemic to the region, the Red List category defined by the criteria was adopted unaltered and if on the other hand, conspecific populations outside the region were judged to experience a "rescue effect" to the target population, the global

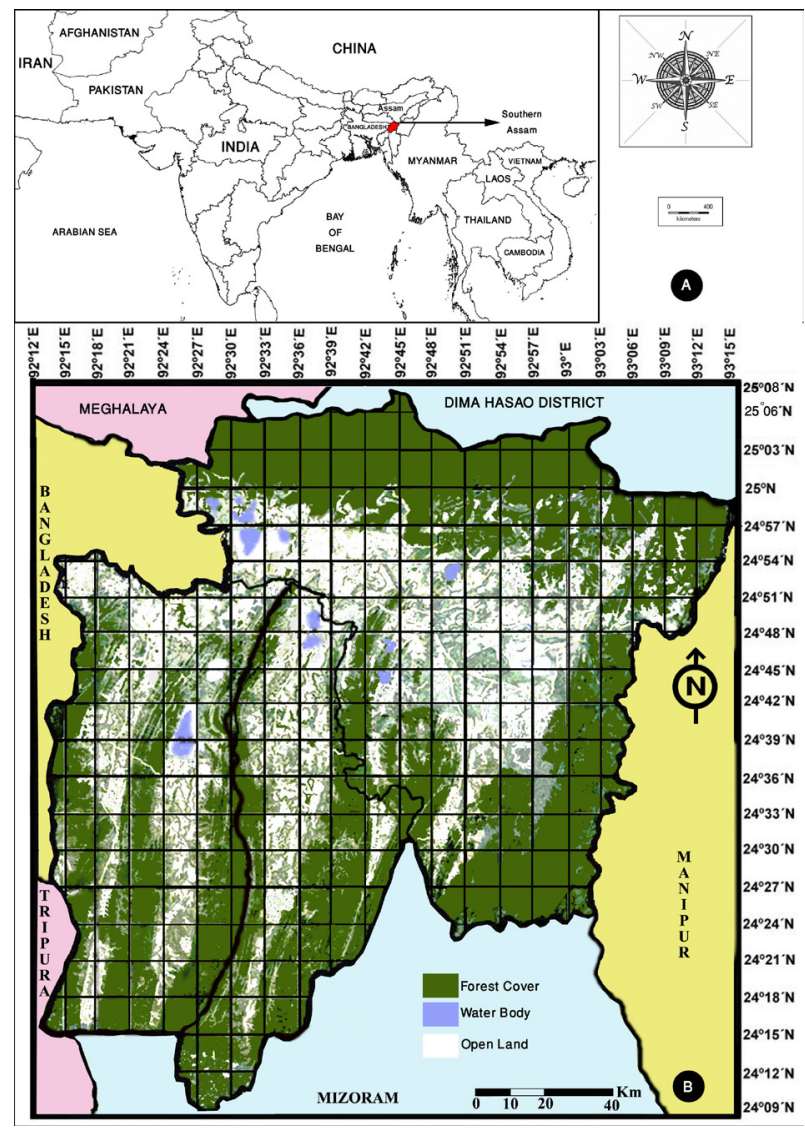

Image 1. A - Map of India showing location of southern Assam. B - Grid map of southern Assam.

assessment was downgraded by one step to fit the IUCN regional Red List guidelines (IUCN 2003), such as changing the category from Endangered (EN) to Vulnerable (VU) or from VU to Near Threatened (NT). Taxa that have been upor downgraded in the regional Red List is clearly indicated by a dot after the category.

Taxa falling under the IUCN Red List Criteria are dealt in detail with - synonyms if any, description, phenology, distribution and specimen(s) examined as well as their status, and criteria for determining the status. Materials cited in Table 1 have been seen and are deposited in the herbarium of Botanical Survey of India, Eastern Regional Centre (ASSAM), unless otherwise stated. Digitalized specimens are cited along with their barcodes.

\section{Results and Discussion}

A total of 90 taxa are recorded in the present study (Table 1), of which genera Ixora, Ophiorrhiza and Psychotria have a maximum number with seven taxa each, followed by Oldenlandia with six taxa, Mussaenda with five taxa, Chassalia and Lasianthus with four taxa each, Tarenna and Uncaria are represented by three taxa each and Cephalanthus, Leptomischus, Mitragyna, 
Table 1. List of Rubiaceae members recorded from southern Assam

\begin{tabular}{|c|c|c|}
\hline & Name of the Species & $\begin{array}{l}\text { Specimen(s) examined / } \\
\text { References }\end{array}$ \\
\hline 1 & Acranthera tomentosa Hook. f. & $\begin{array}{l}\text { De Silva in Wall. Cat. no. } 8398 \\
\text { (K-000030962). }\end{array}$ \\
\hline 2 & Aidia densiflora (Wall.) Masam. & $\begin{array}{l}\text { U.N. Kanjilal 4819, 4852, 4895; } \\
\text { R.N. De } 19165 .\end{array}$ \\
\hline 3 & $\begin{array}{l}\text { Argostemma sarmentosum } \\
\text { Wall. }\end{array}$ & H.A. Barbhuiya 694, 960. \\
\hline 4 & $\begin{array}{l}\text { Benkara fasciculata (Roxb.) } \\
\text { Ridsdale }\end{array}$ & $\begin{array}{l}\text { Kanjilal et al. (1939) as Randia } \\
\text { fasciculata (Roxb.) DC. }\end{array}$ \\
\hline 5 & Canthium glabrum Blume & H.A. Barbhuiya 866 . \\
\hline 6 & $\begin{array}{l}\text { Catunaregam spinosa (Thunb.) } \\
\text { Tirveng. }\end{array}$ & $\begin{array}{l}\text { R.B. Majumdar 73481, } 73271 \text {; } \\
\text { H.A. Barbhuiya } 703 \text {. }\end{array}$ \\
\hline 7 & Cephalanthus occidentalis L. & $\begin{array}{l}\text { Haviland (1897); Kanjilal et al. } \\
\text { (1939). }\end{array}$ \\
\hline 8 & $\begin{array}{l}\text { Cephalanthus tetrandrus } \\
\text { (Roxb.) Ridsdale \& Bakh. f. }\end{array}$ & $\begin{array}{l}\text { Hooker (1880) as Cephalanthus } \\
\text { naucleoides DC. }\end{array}$ \\
\hline 9 & $\begin{array}{l}\text { Ceriscoides campanulata } \\
\text { (Roxb.) Tirveng. }\end{array}$ & $\begin{array}{l}\text { Kanjilal et al. (1939) as } \\
\text { Gardenia campanulata Roxb. }\end{array}$ \\
\hline 10 & $\begin{array}{l}\text { Chassalia curviflora (Wall.) } \\
\text { Thwaites var. ellipsoides } \\
\text { Hook. f. }\end{array}$ & H.A. Barbhuiya 539, 567. \\
\hline 11 & $\begin{array}{l}\text { Chassalia curviflora (Wall.) } \\
\text { Thwaites var. longifolia Hook. f. }\end{array}$ & $\begin{array}{l}\text { R.N. De 21644. Deb \& Krishna } \\
\text { (1982). }\end{array}$ \\
\hline 12 & $\begin{array}{l}\text { Chassalia curviflora (Wall.) } \\
\text { Thwaites var. ophioxyloides } \\
\text { (Wall.) Deb \& B. Krishna }\end{array}$ & H.A. Barbhuiya 704, 775, 959. \\
\hline 13 & $\begin{array}{l}\text { Chassalia staintonii (H.Hara) } \\
\text { Deb \& Mondal }\end{array}$ & H.A. Barbhuiya 701. \\
\hline 14 & $\begin{array}{l}\text { Dentella repens J.R. Forst. \& G. } \\
\text { Forst. var. serpyllifolia (Wall. ex } \\
\text { Craib) Verdc. }\end{array}$ & R.B. Majumdar 83990. \\
\hline 15 & $\begin{array}{l}\text { Discospermum abnorme } \\
\text { (Korth.) S.J. Ali \& Robbr. }\end{array}$ & $\begin{array}{l}\text { U.N. Kanjilal 4741; N.L. Bor } \\
14040 .\end{array}$ \\
\hline 16 & Gardenia coronaria Buch.-Ham. & R.N. De 17596. \\
\hline 17 & Hedyotis scandens Roxb. & $\begin{array}{l}\text { R.B. Majumdar 61059, } 83966, \\
83812 ; \text { H.A. Barbhuiya } 845 .\end{array}$ \\
\hline 18 & $\begin{array}{l}\text { Hymenodictyon orixense } \\
\text { (Roxb.) Mabb. }\end{array}$ & R.N. De 26521. \\
\hline 19 & $\begin{array}{l}\text { Hyptianthera stricta (Roxb.) } \\
\text { Wight \& Arn. }\end{array}$ & R.B. Majumdar 73130. \\
\hline 20 & Ixora acuminata Roxb. & H.A. Barbhuiya 500, 901. \\
\hline 21 & Ixora balakrishnii Deb \& Rout & $\begin{array}{l}\text { R.S. Rao 9058; H.A. Barbhuiya } \\
958 .\end{array}$ \\
\hline 22 & $\begin{array}{l}\text { Ixora finlaysoniana Wall. ex } \\
\text { G. Don }\end{array}$ & $\begin{array}{l}\text { R.S. Rao 9129; U.N. Kanjilal } \\
\text { 4888; H.A. Barbhuiya } 864 .\end{array}$ \\
\hline 23 & $\begin{array}{l}\text { Ixora malabarica (Dennst.) } \\
\text { Mabb. }\end{array}$ & H.A. Barbhuiya 919. \\
\hline 24 & $\begin{array}{l}\text { Ixora nigricans } \mathrm{R} \text {. Br. ex Wight } \\
\text { \& Arn. }\end{array}$ & H.A. Barbhuiya 542. \\
\hline 25 & Ixora rangonensis Bremek. & H.A. Barbhuiya 659. \\
\hline 26 & Ixora subsessilis Wall. ex G. Don & Kanjilal et al. (1939). \\
\hline 27 & Lasianthus attenuatus Jack & R.S. Rao 9082. \\
\hline 28 & Lasianthus cyanocarpus Jack & Kanjilal et al. (1939). \\
\hline 29 & $\begin{array}{l}\text { Lasianthus hirsutus (Roxb.) } \\
\text { Merr. }\end{array}$ & U.N. Kanjilal 4747. \\
\hline 30 & Lasianthus lancifolius Hook.f. & H.A. Barbhuiya 990. \\
\hline 31 & $\begin{array}{l}\text { Leptomischus modesta } \\
\text { (Hook.f.) Deb }\end{array}$ & $\begin{array}{l}\text { R.L. Keenan s.n. (K-000031981, } \\
\text { 000031982). }\end{array}$ \\
\hline 32 & $\begin{array}{l}\text { Leptomischus wallichii (Hook.f.) } \\
\text { H.S. Lo }\end{array}$ & $\begin{array}{l}\text { W. Gomez } 8424 \text { (K-000760568, } \\
\text { 000760569); Hooker (1880) } \\
\text { and Kanjilal et al. (1939) as } \\
\text { Polysolenia wallichii Hook. f. }\end{array}$ \\
\hline 33 & $\begin{array}{l}\text { Metadina trichotoma (Zoll. \& } \\
\text { Moritzi) Bakh. f. }\end{array}$ & $\begin{array}{l}\text { Kanjilal et al. (1939) as Adina } \\
\text { polycephala Benth. }\end{array}$ \\
\hline
\end{tabular}

\begin{tabular}{|c|c|c|}
\hline & Name of the Species & $\begin{array}{l}\text { Specimen(s) examined / } \\
\text { References }\end{array}$ \\
\hline 34 & Meyna spinosa Roxb. ex Link & H.A. Barbhuiya 193. \\
\hline 35 & $\begin{array}{l}\text { Mitragyna diversifolia (Wall. ex } \\
\text { G. Don) Havil. }\end{array}$ & $\begin{array}{l}\text { N.G. Young 13158; R.B. } \\
\text { Majumdar 74216; R.N. De } \\
\text { 20524; U.N. Kanjilal 4673; H.A. } \\
\text { Barbhuiya } 695 .\end{array}$ \\
\hline 36 & $\begin{array}{l}\text { Mitragyna rotundifolia (Roxb.) } \\
\text { Kuntze }\end{array}$ & H.A. Barbhuiya 457. \\
\hline 37 & Morinda angustifolia Roxb. & $\begin{array}{l}\text { R.B. Majumdar 74132, 73286, } \\
\text { 73107; H.A. Barbhuiya 561, } \\
\text { 592, 633, } 652 \text {. }\end{array}$ \\
\hline 38 & Morinda villosa Hook. f. & R.S. Rao 9120. \\
\hline 39 & Mussaenda corymbosa Roxb. & H.A. Barbhuiya 112234. \\
\hline 40 & Mussaenda frondosa $\mathrm{L}$. & R.B. Majumdar 74288, 73330 \\
\hline 41 & Mussaenda glabra Vahl & $\begin{array}{l}\text { R.B. Majumdar 73115, 73480; } \\
\text { U.N. Kanjilal 627P. }\end{array}$ \\
\hline 42 & Mussaenda keenanii Hook. f. & $\begin{array}{l}\text { R.L. Keenan s.n. (K-000032009, } \\
000032010) ; \text { H.A. Barbhuiya } \\
112269 .\end{array}$ \\
\hline 43 & Mussaenda roxburghii Hook. f. & $\begin{array}{l}\text { R.B. Majumdar 73016; H.A. } \\
\text { Barbhuiya 93, 219, 682, 777, } \\
\text { 913. }\end{array}$ \\
\hline 44 & $\begin{array}{l}\text { Mycetia longifolia (Wall.) } \\
\text { Kuntze }\end{array}$ & $\begin{array}{l}\text { R.B. Majumdar 73045, 73126; } \\
\text { H.A. Barbhuiya } 774 .\end{array}$ \\
\hline 45 & $\begin{array}{l}\text { Mycetia mukerjiana Deb \& } \\
\text { R.M.Dutta }\end{array}$ & H.A. Barbhuiya 773. \\
\hline 46 & $\begin{array}{l}\text { Myrioneuron nutans Wall. } \\
\text { ex Kurz }\end{array}$ & $\begin{array}{l}\text { R.B. Majumdar } 73126,83972 ; \\
\text { H.A. Barbhuiya } 772 \text {. }\end{array}$ \\
\hline 47 & Nauclea orientalis (L.) L. & U.N. Kanjilal 4810. \\
\hline 48 & $\begin{array}{l}\text { Neolamarckia cadamba (Roxb.) } \\
\text { Bosser }\end{array}$ & H.A. Barbhuiya 778. \\
\hline 49 & $\begin{array}{l}\text { Neonauclea purpurea (Roxb.) } \\
\text { Merr. }\end{array}$ & P.C. Kanjilal 9633. \\
\hline 50 & $\begin{array}{l}\text { Neonauclea sessilifolia (Roxb.) } \\
\text { Merr. }\end{array}$ & R.N. De 20784. \\
\hline 51 & $\begin{array}{l}\text { Nostolachma khasiana (Korth.) } \\
\text { Deb \& J. Lahiri }\end{array}$ & R.B. Majumdar 74121, 84268. \\
\hline 52 & $\begin{array}{l}\text { Oldenlandia auricularia (L.) K. } \\
\text { Schum. }\end{array}$ & R.B. Majumdar 73155, 83986. \\
\hline 53 & $\begin{array}{l}\text { Oldenlandia cristata (Willd. ex } \\
\text { Roem. \& Schult.) ined. }\end{array}$ & $\begin{array}{l}\text { Hooker (1880); Kanjilal et al. } \\
\text { (1939) as Hedyotis vestita R. Br. } \\
\text { ex G. Don. }\end{array}$ \\
\hline 54 & $\begin{array}{l}\text { Oldenlandia diffusa (Willd.) } \\
\text { Roxb. }\end{array}$ & $\begin{array}{l}\text { R.B. Majumdar 73305, 73488, } \\
74190 .\end{array}$ \\
\hline 55 & $\begin{array}{l}\text { Oldenlandia glabra (Roxb.) } \\
\text { Kuntze }\end{array}$ & $\begin{array}{l}\text { Hooker (1880) as Hedyotis } \\
\text { glabra (Roxb.) R. Br. ex Wall. }\end{array}$ \\
\hline 56 & $\begin{array}{l}\text { Oldenlandia tenelliflora } \\
\text { (Blume) Kuntze }\end{array}$ & H.A. Barbhuiya 170. \\
\hline 57 & Oldenlandia verticillata $\mathrm{L}$. & R.B. Majumdar 84242 . \\
\hline 58 & Ophiorrhiza hispida Hook. f. & R.B. Majumdar 73057, 73429. \\
\hline 59 & Ophiorrhiza mungos L. & R.B. Majumdar 73161 \\
\hline 60 & Ophiorrhiza nutans C.B. Clarke & H.A. Barbhuiya 539. \\
\hline 61 & Ophiorrhiza ochroleuca Hook. f. & $\begin{array}{l}\text { Hooker (1880); Kanjilal et al., } \\
\text { (1939); Kar \& Panigrahi (1963). }\end{array}$ \\
\hline 62 & $\begin{array}{l}\text { Ophiorrhiza repens (Wall. ex } \\
\text { G.Don) Bennet }\end{array}$ & $\begin{array}{l}\text { R.B. Majumdar 73181; H.A. } \\
\text { Barbhuiya } 914 .\end{array}$ \\
\hline 63 & $\begin{array}{l}\text { Ophiorrhiza rugosa Wall. var. } \\
\text { argentea (Wall. ex G.Don) Deb } \\
\text { \& Mondal }\end{array}$ & H.A. Barbhuiya 865 . \\
\hline 64 & $\begin{array}{l}\text { Ophiorrhiza succirubra King ex } \\
\text { Hook. f. }\end{array}$ & $\begin{array}{l}\text { H.A. Barbhuiya 568, } 915 \text {; P.S. } \\
\text { Das s.n. }\end{array}$ \\
\hline 65 & $\begin{array}{l}\text { Oxyceros longiflorus (Lam.) } \\
\text { T.Yamaz. }\end{array}$ & $\begin{array}{l}\text { Kanjilal et al. (1939) as Randia } \\
\text { longiflora Lam. }\end{array}$ \\
\hline 66 & Paederia foetida $\mathrm{L}$. & P.S. Das s.n. \\
\hline
\end{tabular}




\begin{tabular}{|c|c|c|}
\hline & Name of the Species & $\begin{array}{l}\text { Specimen(s) examined / } \\
\text { References }\end{array}$ \\
\hline 67 & Pavetta indica $\mathrm{L}$. & R.B. Majumdar 74123, 74136. \\
\hline 68 & $\begin{array}{l}\text { Prismatomeris albidiflora } \\
\text { Thwaites }\end{array}$ & R.B. Majumdar 73115. \\
\hline 69 & $\begin{array}{l}\text { Prismatomeris tetrandra } \\
\text { (Roxb.) K. Schum }\end{array}$ & H.A. Barbhuiya 702, 707. \\
\hline 70 & Psychotria adenophylla Wall. & Hooker (1880). \\
\hline 71 & Psychotria calocarpa Kurz & H.A. Barbhuiya 705. \\
\hline 72 & Psychotria denticulata Wall. & R.B. Majumdar 83808. \\
\hline 73 & $\begin{array}{l}\text { Psychotria erratica Hook. f. var. } \\
\text { erratica }\end{array}$ & H.A. Barbhuiya 771. \\
\hline 74 & Psychotria monticola Kurz & $\begin{array}{l}\text { R.B. Majumdar 73108, 73117, } \\
\text { 73479, 83946; H.A. Barbhuiya } \\
660,706,776,994 \text {. }\end{array}$ \\
\hline 75 & $\begin{array}{l}\text { Psychotria silhetensis Hook. f. } \\
\text { subsp. silhetensis }\end{array}$ & H.A. Brbhuiya 855 . \\
\hline 76 & Psychotria symplocifolia Kurz & $\begin{array}{l}\text { R.B. Majumdar } 8422 ; \text { H.A. } \\
\text { Barbhuiya } 484 .\end{array}$ \\
\hline 77 & $\begin{array}{l}\text { Psydrax dicoccos Gaertn. var. } \\
\text { dicoccos }\end{array}$ & U.N. Kanjilal 4923. \\
\hline 78 & Richardia scabra L. & $\begin{array}{l}\text { R.B. Majumdar 73367, 73495, } \\
\text { 74210, } 8430 \text { A. }\end{array}$ \\
\hline 79 & $\begin{array}{l}\text { Saprosma ternata (Wall.) } \\
\text { Hook. f. }\end{array}$ & N.L. Bor 13948, 14030. \\
\hline 80 & Silvianthus bracteatus Hook. f. & H.A. Barbhuiya 770. \\
\hline 81 & Spermacoce hispida L. & $\begin{array}{l}\text { R.B. Majumdar 73099; H.A. } \\
\text { Barbhuiya } 13 .\end{array}$ \\
\hline 82 & Spiradiclis bifida Kurz & $\begin{array}{l}\text { Hooker (1880); Kanjilal et al., } \\
\text { (1939); Kar \& Panigrahi (1963). }\end{array}$ \\
\hline 83 & $\begin{array}{l}\text { Tarenna asiatica (L.) Kuntze ex } \\
\text { K.Schum. }\end{array}$ & R.B. Majumdar 84221. \\
\hline 84 & $\begin{array}{l}\text { Tarenna campaniflora (Hook.f.) } \\
\text { N.P. Balakr. }\end{array}$ & $\begin{array}{l}\text { U.N. Kanjilal 4724; H.A. } \\
\text { Barbhuiya } 112233 .\end{array}$ \\
\hline 85 & $\begin{array}{l}\text { Tarenna odorata (Roxb.) B.L. } \\
\text { Rob. }\end{array}$ & $\begin{array}{l}\text { R.B. Majumdar } 83944 ; \text { H.A. } \\
\text { Barbhuiya } 857 .\end{array}$ \\
\hline 86 & $\begin{array}{l}\text { Tarennoidea wallichii (Hook. f.) } \\
\text { Tirveng. \& Sastre }\end{array}$ & $\begin{array}{l}\text { U.N. Kanjilal 4798, 4815; H.A. } \\
\text { Barbhuiya } 1005 .\end{array}$ \\
\hline 87 & Uncaria macrophylla Wall. & $\begin{array}{l}\text { Hooker (1880); Haviland } \\
\text { (1897). }\end{array}$ \\
\hline 88 & Uncaria scandens (Sm.) Hutch. & R.B. Majumdar 73070. \\
\hline 89 & Uncaria sessilifructus Roxb. & $\begin{array}{l}\text { Haviland (1897); Brandis } \\
\text { (1906). }\end{array}$ \\
\hline 90 & $\begin{array}{l}\text { Wendlandia paniculata (Roxb.) } \\
\text { DC. }\end{array}$ & Kanjilal et al. (1939). \\
\hline
\end{tabular}

Morinda, Mycetia, Neonauclea and Prismatomeris are represented by two species each. Whereas other genera, viz., Acranthera, Aidia, Argostemma, Benkara, Canthium, Catunaregam, Ceriscoides, Dentella, Discospermum, Gardenia, Hedyotis, Hymenodictyon, Hyptianthera, Metadina, Meyna, Myrioneuron, Nauclea, Neolamarckia, Nostolachma, Oxyceros, Paederia, Pavetta, Psydrax, Richardia, Saprosma, Silvianthus, Spermacoce, Spiradiclis, Tarennoidea, and Wendlandia are represented by only one taxon each. Of these, 14 taxa, representing $16 \%$, are found to be threatened and categorized under various IUCN Categories (one probably Extinct, one Regionally Extinct, two Critically Endangered, five Endangered, three Vulnerable and two Near Threatened) of which four taxa are endemic to northeastern India. Leptomischus modesta
(Hook.f.) Deb is endemic to Cachar District; whereas Chassalia curviflora (Wall.) Thwaites var. ellipsoides Hook. f., Mussaenda keenanii Hook. f. and Leptomischus wallichii (Hook. f.) H.S. Lo are restricted to Assam and Meghalaya only. Mussaenda corymbosa Roxb. is reported for the first time from northeastern India and Chassalia curviflora (Wall.) Thwaites var. ellipsoides Hook. f. and Chassalia staintonii (H.Hara) Deb \& Mondal are reported for the first time from Assam based on our collections. Chassalia curviflora var. ellipsoides and Mussaenda keenanii are rediscovered after a gap of ca. 140 years; present collections of both the taxa also constitute their second authentic collection after type.

\section{Enumeration of threatened and endemic taxa}

1. Acranthera tomentosa Hook.f., Fl. Brit. India 3: 92. 1880; Kanjilal et al., Fl. Assam 3: 48. 1939.

Epiphyte, hirsutely tomentose. Stem stout. Leaves elliptic or oblanceolate, $10-25 \times 5.2-7.5 \mathrm{~cm}$, acute at apex, hirsute; petiole $2-2.5 \mathrm{~cm}$ long, hirsute. Flowers bluish, in subterminal bracteate cymes. Calyx tube oblong; lobes 5, linear-lanceolate, $1.5-2.5 \mathrm{~cm}$ long. Corolla villous; tube funnel-shaped, $3.5-4 \mathrm{~cm}$ long; lobes 5, short, rounded. Stamens 5, included; filaments filiform; anthers cohering into a tube, pointed and spurred at their tips. Ovary fusiform, 1-celled; ovules numerous, on two parietal 2-lamellate placenta; style filiform; stigma clavate, furrowed. Berry ca. $3.8 \mathrm{~cm}$ long with persistent calyx lobes.

Flowering \& Fruiting: July-October.

Distribution: India [Assam (Cachar), Arunachal Pradesh, Meghalaya, Nagaland]; Bangladesh.

Specimen examined: Nil.

Global Red List Category: Vulnerable (Walter \& Gillett 1998).

Regional Red List Category and Criteria: The species was collected by R.L. Keenan from Cachar District of Assam more than 140 years ago. During the present study the entire southern Assam was surveyed, but it could not be located. Therefore, following IUCN Red List Categories and Criteria (IUCN 2001, 2003), it has been categorized as Regionally Extinct (RE).

2. Chassalia curviflora (Wall.) Thwaites var. ellipsoides Hook. f., Fl. Brit. India 3: 177. 1880; Deb \& B. Krishna in Bull. Bot. Surv. India 24: 223. 1982. (Image 2(A)).

Shrub, upto $1.5 \mathrm{~m}$ high. Leaves broadly ellipticlanceolate, 10-18 × 3-6 cm, membranous, acuminate at apex, sub-acute at base, glabrous; lateral nerves 7-12 pairs, prominent on lower surface; petiole slender, 1-2 $\mathrm{cm}$ long. Stipules deciduous, deltoid, 2.5-3.5 mm long. Inflorescence terminal cymes, $3-5 \mathrm{~cm}$ long. Pedicels short. 
Calyx tube 3-4 mm long, whitish; lobes 5, pinkish, short deltoid. Corolla pinkish-white outside; tube $1.8-2.3 \mathrm{~cm}$ long, curved, yellow inside; lobes 5, triangular, 2-3 mm long, incurved, white inside. Fruits blackish, ellipsoid, ca. $10 \times 7 \mathrm{~mm}$, smooth.

Flowering \& Fruiting: April-November.

Distribution: India [Assam (Cachar-present record), Meghalaya], endemic to northeastern India.

Specimen examined: 567 (ASSAM), 25.iv.2011, Assam, Cachar, Borail Wildlife Sanctuary, West Block, Near Malidar, coll. H.A. Barbhuiya.

Global Red List Category: Not Evaluated.

Regional Red List Category and Criteria: The taxon was known only from its type collection and the present collection is made after 140 years. Extent of occurrence is estimated to be ca. $40 \mathrm{~km}^{2}$ (Criteria EN B1 $<5,000 \mathrm{~km}^{2}$ ); The area of occupancy is calculated to be ca. $20 \mathrm{~km}^{2}$ (Criteria EN B2 $<500 \mathrm{~km}^{2}$ ), while there is a continuous decline in area of occupancy due to slash-and-burn cultivation and tree felling (Criteria EN B2b(ii)). Following IUCN Red List Categories and Criteria (IUCN 2001), this taxon has been classified as Endangered (EN). As there is no possibilities of rescue from neighboring regions, hence there is no change to the initial assessment.

Note: This taxon was first described by Hooker (1880) on the basis of a collection made by C.B. Clarke in 1872 from Jarain area in Jaintia Hills of Meghalaya. Thereafter this variety was not reported to be collected by anyone. Hence, the present collection made after 140 years is a new record for the state of Assam.

3. Chassalia staintonii (H.Hara) Deb \& Mondal in J. Jap. Bot. 57(5): 160. 1982; S.N. Das \& S.C. Roy in J. Econ. Taxon. Bot. 5(2): 473. 1984. Ophiorrhiza staintonii H.Hara in J. Jap. Bot. 52(12): 358. 1977. Image 2(B).

Erect shrub, ca. $1 \mathrm{~m}$ tall. Leaves oblong-lanceolate, 16-24 × 3.6-5.2 cm, glabrous, base cuneate, abruptly acuminate at apex; lateral nerves 11-14 on either side of midvein; petiole $1.5-3 \mathrm{~cm}$ long. Stipules ovate-triangular with bifid apex. Inflorescence terminal or axillary, many flowered cymes. Flowers whitish-pink, 5-merous. Calyx 0.5-1 mm long, with prominent nerves; lobes 5 , subulate. Corolla tubular, 12-15 mm long; lobes 5, ca. $3 \mathrm{~mm}$ long with incurved apex. Stamens 5; filaments $2-3 \mathrm{~mm}$ long; anthers 3-4 $\mathrm{mm}$ long. Ovary 2-locular, 1-ovule in each locule; style 5-15 mm long; stigma bifid.

Flowering \& Fruiting: May-November.

Distribution: India [Assam (Cachar-present record), Sikkim, West Bengal]; Nepal.

Specimen examined: 701 (ASSAM), 08.x.2011, Assam, Cachar, Borail Wildlife Sanctuary, West Block, Near
Kalaincherra, coll. H.A. Barbhuiya.

Global Red List Category: Not Evaluated.

Regional Red List Category and Criteria: The area of occupancy is ca. $505 \mathrm{~km}^{2}$ (Criteria VU B2<2,000 km²), currently known from about six locations (Criteria VU $B 2<10$ ), while there is a continuous decline in area of occupancy due to slash-and-burn cultivation and tree felling (Criteria VU B2b(ii)). Following IUCN Red List Categories and Criteria (IUCN 2001), this taxon has been classified as Vulnerable (VU). As there is are possibilities of rescue from neighboring regions, hence there is no change to the initial assessment.

Note: This species was first described by Hara (1977) as Ophiorrhiza staintonii on the basis of a collection made by J.D.A. Stainton from Sikkim and easterm Nepal in 1966 and 1971 respectively. Das \& Roy (1984) reported this species from Jalpaiguri District of West Bengal. It is reported here for the first time from Assam.

4. Ixora malabarica (Dennst.) Mabb. in Taxon 26: 539. 1977; T. Hussain \& S.R. Paul in J. Econ. Taxon. Bot., Addit. Ser. 6. 144. 1989. Chiococca malabarica Dennst., Schlüssel Hortus Malab. 37. 1818. Ixora lanceolaria Colebr., FI. Ind. 1: 397. 1820. Image 2(C).

Small tree, ca. $6 \mathrm{~m}$ tall. Branches slender, pendulous. Leaves linear-lanceolate, $20-22 \times 3.5-4.5 \mathrm{~cm}$, acuminate at apex, somewhat glaucous above, pale beneath; lateral nerves usually $14-20$ pairs; petiole short $0.8-1.3 \mathrm{~cm}$ long. Stipules adpressed, lanceolate, 6-7 mm long. Bracts supporting inflorescence sessile, ovate, 2-2.5 × 1.2-1.5 $\mathrm{cm}$, sub-acute at apex, cuneate at base. Cymes peduncled, trichotomous, pendulous, terminal panicle brachiate, pubescent; bracts linear-lanceolate. Pedicels ternate, 1-2 $\mathrm{mm}$ long, pubescent especially at base. Flower not seen. Fruits greenish turn to reddish, globose, 5-8 × 9-10 mm, smooth, crowned with subulate erect calyx teeth.

Fruiting: June.

Distribution: India [Assam (Cachar), Meghalaya, West Bengal, Maharashtra, Karnataka, Tamil Nadu, Goa, Kerala]; Philippines?

Specimen examined: 919 (Assam), 04.vi.2012, Assam, Cachar, Borail Wildlife Sanctuary, East Block, Nimatra Pahar, coll. H.A. Barbhuiya.

Global Red List Category: Vulnerable (IUCN 2012).

Regional Red List Category and Criteria: The Extent of occurrence is estimated to be ca. $25 \mathrm{~km}^{2}$ (Criteria EN $\left.\mathrm{B} 1<5,000 \mathrm{~km}^{2}\right)$. The area of occupancy is calculated to be ca. $11 \mathrm{~km}^{2}$ (Criteria EN B2<500 $\mathrm{km}^{2}$ ) and there is only one known locality (Criteria EN B1a $\leq 5$ ), while there is a continuous decline in area of occupancy due to encroachment of National Highway 54 (Criteria EN B2b(ii)). 

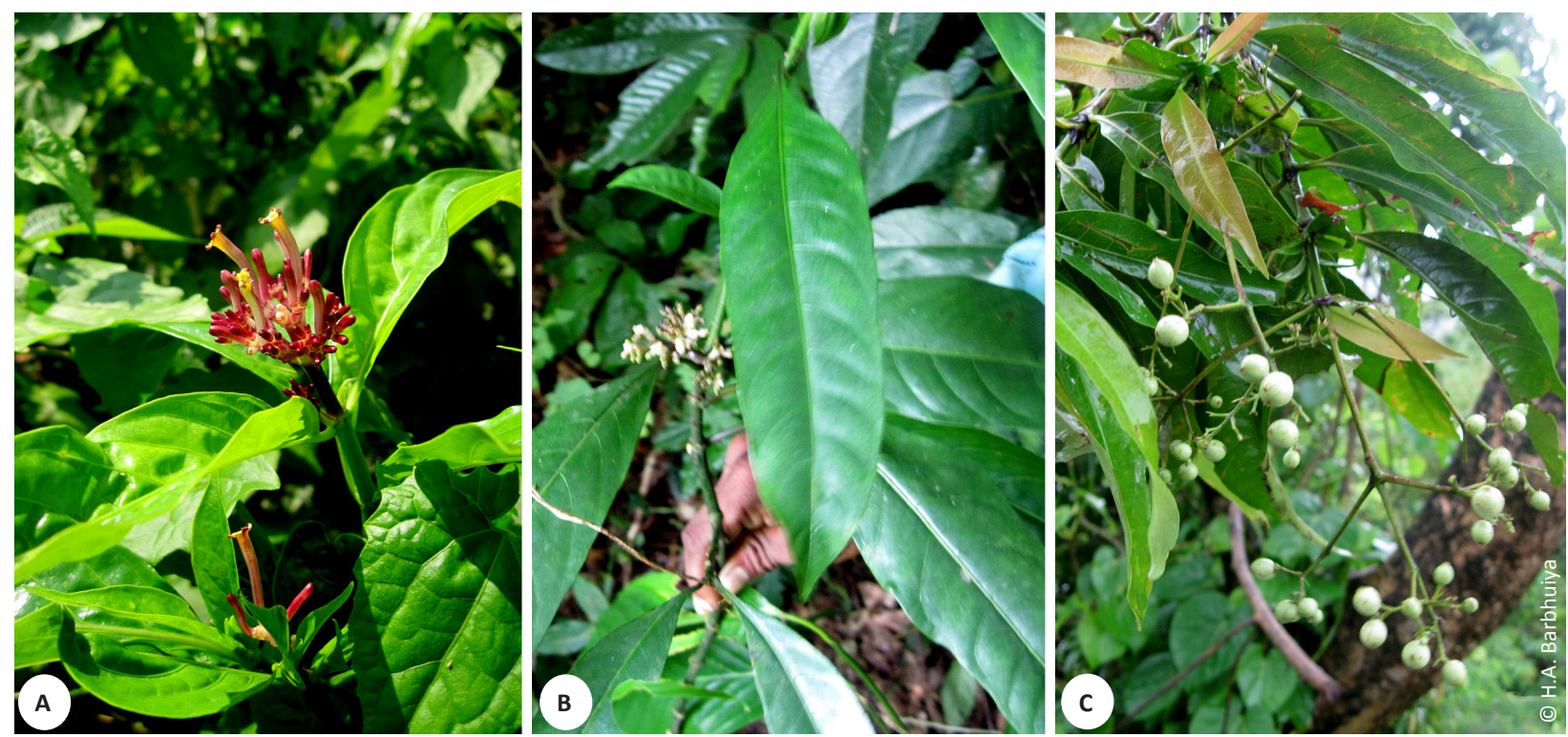

Image 2. A - Chassalia curviflora (Wall.) Thwaites var. ellipsoides Hook. f.; B - Chassalia staintonii (H.Hara) Deb \& Mondal.; C - Ixora malabarica (Dennst.) Mabb.

Following IUCN Red List Categories and Criteria (IUCN 2001), the species has been classified as Endangered (EN). As there is no possibilities of rescue from neighboring regions, hence there is no change to the initial assessment.

5. Ixora rangonensis Bremek. in Indian Forester 85: 374. 1959; Barbhuiya et al. in Taiwania 57(4): 413. 2012. Image 2(D).

Shrub up to $1 \mathrm{~m}$ high. Leaves oblanceolate, $20-29 \times$ $8-9.5 \mathrm{~cm}$, cuneate at base, caudate at apex, cauda ca. $2.5 \mathrm{~cm}$ long, entire, glabrous on both sides; lateral nerves 11-13 pairs; petiole 1-1.2 cm long. Stipules linear-triangular, ca. $1.5 \mathrm{~cm}$ long. Bracts supporting inflorescence sessile, ovate, $4-4.4 \times 1.8-2.1 \mathrm{~cm}$, base cordate, apex acuminate, nerves 8-9 on either side of midrib. Inflorescence cymose, ca. $10 \mathrm{~cm}$ long, numerous flowered, peduncle $6-8 \mathrm{~cm}$ long, pubescent; bracts linear-lanceolate, 5-6 mm long, pubescent. Flowers sessile to pedicellate; pedicels to $2 \mathrm{~mm}$ long, hispid. Calyx tube ca. $2 \mathrm{~mm}$ long; lobes 4 , light brown, ovate, 1-2 mm, pubescent. Corolla white, tube 20-25 $\mathrm{mm}$ long, glabrous; lobes 4, oblong, 4.5-5 × 1-1.2 mm, rounded at apex. Stamens 4, 0.6-1 mm long, glabrous; anthers sub-basifixed. Ovary 2-celled, one ovule in each locule; style slender, as long as corolla tube. Fruit not seen.

Flowering: April-May.

Distribution: India [Assam (Cachar)]; Myanmar.

Specimen examined: 659 (ASSAM), 09.v.2011, Assam, Cachar, Borail Wildlife Sanctuary, Kalain Range, Near Kalaincherra, coll. H.A. Barbhuiya.

Global Red List Category: Not Evaluated.
Regional Red List Category and Criteria: The Extent of occurrence is estimated to be ca. $15 \mathrm{~km}^{2}$ (Criteria CR $\left.\mathrm{B} 1<100 \mathrm{~km}^{2}\right)$; The area of occupancy is estimated to be $8 \mathrm{~km}^{2}$ (Criteria CR B2<10 $\mathrm{km}^{2}$ ), known to exist at only a single location (Criteria CR B2a). The population size is estimated to number less than 10 individuals and so far only one plant was seen (Criteria CR D $<50$ mature individual), while there is degradation of the habitat (Criteria CR B2b(iii)) due to slash-and-burn cultivation and tree felling. Following the IUCN Red List Categories and Criteria (IUCN 2001), this species has been classified as Critically Endangered (CR). As there is no possibilities of rescue from neighboring regions, hence there is no change to the initial assessment.

6. Leptomischus modesta (Hook. f.) Deb in Phytotaxonomy 1: 16. 2001. Keenania modesta Hook.f., Fl. Brit. India 3: 101. 1880; Kanjilal et al., Fl. Assam 3: 50. 1939.

Low sub-herbaceous herb; branches without spongy swollen corky bark. Leaves opposite, elongate-lanceolate, $15-31 \times 3.8-7.6 \mathrm{~cm}$, abruptly acuminate, membranous, without marginal stalked glands, puberulous beneath; lateral nerves 15-20 on either side; base narrowed into petiole that is $2-2.5 \mathrm{~cm}$ long. Stipules somewhat recurved, membranous, entire, $1.3-2.5 \mathrm{~cm}$ long without marginal stalked glands, Inflorescence in terminal heads. Heads globose, nodding, ca. $2.5 \mathrm{~cm}$. Peduncle short. Flower sessile, heterostylous. Calyx 5 or 6-lobed, ca. $0.7 \mathrm{~cm}$, longer than corolla. Corolla 5-lobed; tube inflated, 
glabrous, throat with a ring of stiff hairs. Stamens 5. Ovary 2-loculed, ovules 8.; style short; stigmas 2, flat. Fruits not seen.

\section{Flowering: April.}

Distribution: India [Assam (Cachar)], Endemic.

Specimen examined: Nil.

Global Red List Category: Not Evaluated.

Regional Red List Category and Criteria: The species is presumed to be Extinct (EX) because an exhaustive survey in the type locality by D.B. Deb in October 1995 (Deb 2001) and by the authors from 2010-2013 to relocate the plant in its type locality or anywhere in the Barak valley region proved futile. The type specimens were collected before the establishment of tea gardens, which might have been the cause of its extinction.

7. Leptomischus wallichii (Hook.f.) H.S. Lo in Acta Phytotax. Sin. 31: 275. 1993. Polysolenia wallichii Hook.f., Gen. Pl. 2: 68. 1873 \& Fl. Brit. India 3: 94. 1880; Kanjilal et al., Fl. Assam 3: 49. 1939. Indopolysolenia wallichii (Hook. f.) Bennet in Indian Forester 107: 437. 1981.

Erect glabrous undershrub. Stem cylindric, woody, simple or branched, glabrous or pubescent above. Leaves lanceolate, 7.6-12.7 cm long, acuminate, pubescent; nerves slender; base narrowed into a petiole, 0.5-2.5 $\mathrm{cm}$ long. Stipules $0.7-1.3 \mathrm{~cm}$ long. Flowers in solitary terminal peduncled heads, ca. $1.2 \mathrm{~cm}$ diam. Peduncle $0.7-2.5 \mathrm{~cm}$ long, slender, pubescent; bracts few, irregular, ovate or linear-oblong ca. $0.4 \times 0.3 \mathrm{~cm}$. Flowers subsessile, 5-merous; pedicel up to $0.2 \mathrm{~cm}$, pubescent; hypanthium ca. $0.2 \times 0.2 \mathrm{~cm}$, obovoid, pubescent. Calyx-tube short; lobes 5 , broadly ovate. Corolla tube $2.2-3 \mathrm{~cm}$ long, slender, pubescent outside, villous inside; lobes 5, ca. $0.2 \times 0.1 \mathrm{~cm}$, ovate-triangular, acute at apex, pubescent. Stamens 5 , attached at middle of corolla tube; filaments ca. $0.2 \mathrm{~cm}$, stout, glabrous; anthers ca. $0.2 \mathrm{~cm}$, dorsifixed, 2-loculed, pubescent. Ovary 2-loculed; ovules many, on peltate placenta; style filiform; stigma short, dilated; Capsules hemispherical, $0.3-0.4 \times 0.3-0.5 \mathrm{~cm}$, membranous, pubescent; seeds many, obovate-triangular, sparsely pubescent.

Flowering \& Fruiting: June-December.

Distribution: India [Assam (Cachar), Meghalaya], Endemic.

Specimen examined: Nil.

Global Red List Category: Rare (Walter \& Gillett, 1998).

Regional Red List Category and Criteria: The Extent of occurrence is estimated to be ca. $35 \mathrm{~km}^{2}$ (Criteria EN $\left.\mathrm{B} 1<5,000 \mathrm{~km}^{2}\right)$; The area of occupancy is calculated to be ca. $15 \mathrm{~km}^{2}$ (Criteria EN B2 $<500 \mathrm{~km}^{2}$ ), known to exist from two locations (Criteria EN B2a $\leq 5$ ), the area of occupancy is rapidly decreasing due to slash-and-burn cultivation (Criteria EN B2b(ii)). Following IUCN Red List Categories and Criteria (IUCN 2001), the species has been classified as Endangered (EN). As there is no possibilities of rescue from neighboring regions, hence there is no change to the initial assessment.

Notes: Leptomischus wallichii (Hook.f.) H.S. Lo was established by Hooker in 1873 as Polysolenia wallichii on the basis of a collection made by W. Gomez from Khasia Mountains, in the mean time R.L. Keenan also collected the plant from Cachar District of Assam. Later in 1968,
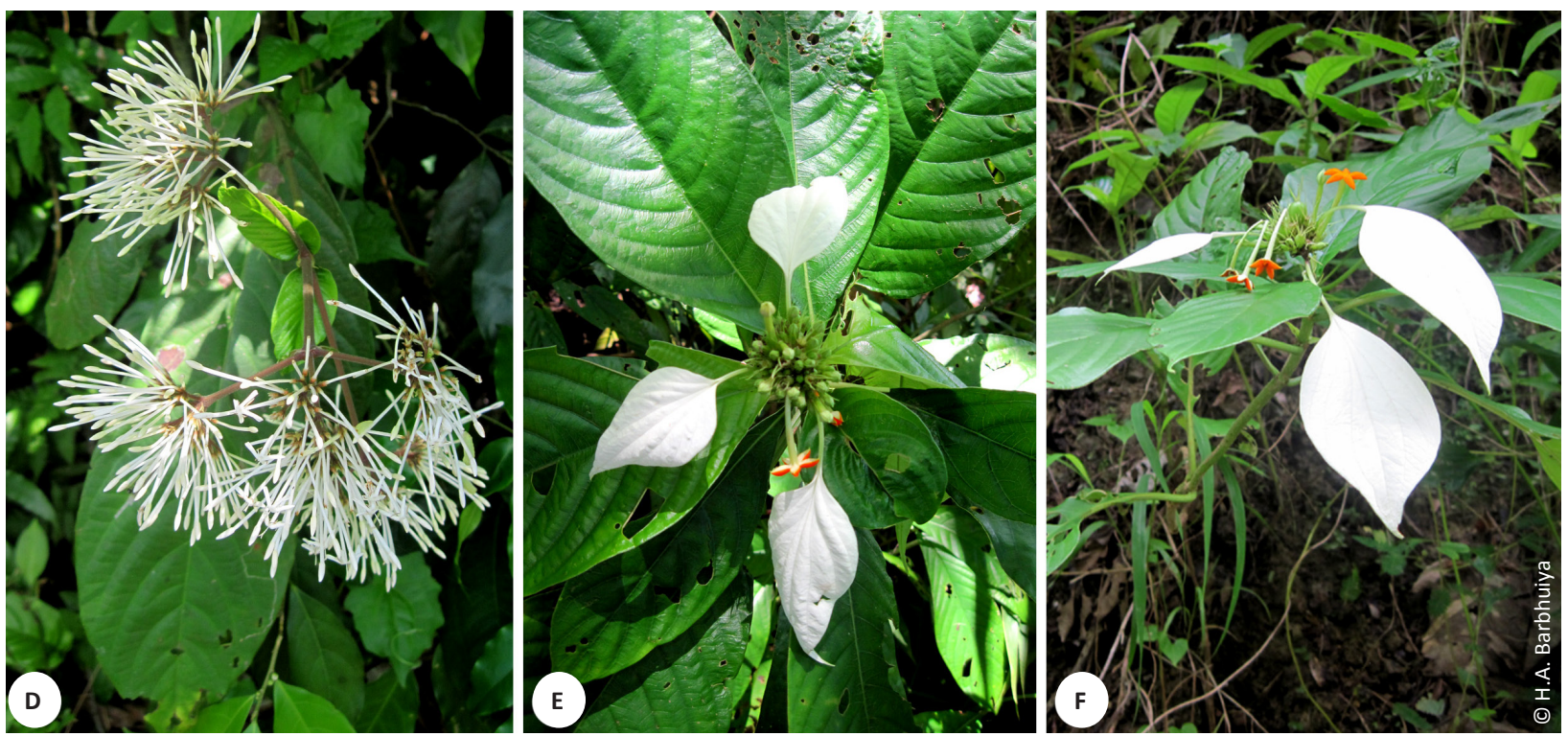

Image 2. D - Ixora rangonensis Bremek.; E - Mussaenda corymbosa Roxb.; F - Mussaenda keenanii Hook. f. 
Balakrishnan collected the plant from Sohha-Dawki area of Meghalaya.

8. Mussaenda keenanii Hook.f., Fl. Brit. India 3: 87. 1880; Kanjilal et al., FI. Assam 3: 45. 1939. Image 2(F).

Erect robust undershrub; stem greenish-white with thick coating of cork, which splits longitudinally. Leaves obovate or broadly ovate, $10-30 \times 5-15 \mathrm{~cm}$, entire, abruptly acuminate, base narrowed into petiole, subcoriaceous, densely tomentose beneath; lateral nerves 15-18 on either side, sub-parallel, very prominent underneath; secondary nerves more or less sub-parallel; petiole $0.5-2.5 \mathrm{~cm}$ long, stout. Stipules broadly ovate acuminate. Cymes, $5-7.6 \mathrm{~cm}$ in across, almost capitate. Calyx 5-parted; teeth filiform ca. $1.3 \mathrm{~cm}$ long, persistent. Corolla ca. $2.5 \mathrm{~cm}$, glabrous, tube slender; lobes 5, small, deep orange, ovate-lanceolate, acuminate. Berry whitish, succulent.

Flowering \& Fruiting: August-January.

Distribution: India [Assam (Cachar, Karimganj), Meghalaya?], Endemic.

Specimen examined: 112269 (ASSAM), 19..viii.2013, Assam, Karimganj, Patheria R.F., coll. H.A. Barbhuiya.

Global Red List Category: Not Evaluated

Regional Red List Category and Criteria: The extent of occurrence is calculated to be ca. $50 \mathrm{~km}^{2}$ (Criteria CR $\left.\mathrm{B} 1<100 \mathrm{~km}^{2}\right)$; area of occupancy is estimated to be ca. $2.2 \mathrm{~km}^{2}$ (Criteria CR B2 $<10 \mathrm{~km}^{2}$ ), currently known to exist from a single location (Criteria CR B2a) and the area of occupancy is decreased due to the establishment of Gopal Tilla T.E., Kalain, Cachar, Assam (Criteria CR B2b(ii)). Following the IUCN Red List Categories and Criteria (IUCN 2001), this species has been classified as Critically Endangered (CR). As there is no possibilities of rescue from neighboring regions, hence there is no change to the initial assessment.

Note: This taxon was first described by Hooker (1880) on the basis of a collection made by R.L. Keenan in 1873 from Gopal Tilla in Cachar District of Assam. Thereafter there are no reports that this species has been collected by anyone. Though, Kanjilal et al. (1939) in "Flora of Assam" mentioned its occurrence from Nongkhlaw area in the Khasi Hills of Meghalaya, no herbarium specimen has been found deposited in any herbaria. Hence, the present collection constitutes its rediscovery after 140 years and may also constitute its second authentic report after its type collection.

9. Mycetia mukerjiana Deb \& R.M. Dutta in Indian Forester 91: 272. 1965; S.C. Das \& M.A. Rahman in Bangladesh J. Bot. 39(2): 218. 2010. Image 2(G).
Small shrub. Leaves broadly elliptic, 25-27.5 × 7.5-9 $\mathrm{cm}$, entire, acuminate, membranous glabrous; lateral nerves 15-20 on either side, alternate, subparallel; petiole 2-3 cm long. Inflorescence on leafless nodes. Flowers pedicellate, bisexual, regular, ca. $10 \mathrm{~mm}$ long; pedicel ca. $4 \mathrm{~mm}$, stout. Calyx tube short, hemispheric; lobes 5 , persistent, chartaceous, 8-11.2 × 2-2.5 mm, lanceolate, acuminate, distinctly veined, scattered tomentose. Corolla yellow, fleshy, tube slightly longer than calyx lobes; teeth 5 , short, reduplicate valvate, hispidulous at back, long pubescent within. Stamens 5, epipetalous, included within tube; filaments about $4-5 \mathrm{~mm}$, flattened, adhering to corolla tube, slightly free above, villous below; anthers oblong ca. $3 \mathrm{~mm}$ long, dorsifixed, puberulous, 2-lobed; lobes parallel, longitudinally dehiscent. Carpels 2, syncarpous; ovary inferior, 1.5-2 mm long, hemispheric, hispidulous, 2 chambered. Fruits small hemispheric berry, 2 chambered; seeds blackish minute, angled.

Flowering \& Fruiting: August-December.

Distribution: India [Assam (Cachar, Lakhimpur), Mizoram, Nagaland]; Bangladesh.

Specimen examined: 778 (ASSAM), 10.xi.2011, Assam, Cachar, Borail Wildlife Sanctuary, West Block, Kalain Range, 15 No. Hill, coll. H.A. Barbhuiya.

Global Red List Category: Indeterminate (Walter \& Gillett 1998).

Regional Red List Category and Criteria: The area of occupancy is calculated to be ca. $502 \mathrm{~km}^{2}$ (Criteria VU $\mathrm{B} 2<2,000 \mathrm{~km}^{2}$ ), currently known to exist in 5-6 locations (Criteria VU $\mathrm{B} 2 \mathrm{a} \leq 10$ ). The area of occupancy is rapidly decreasing due to slash-and-burn cultivation (Criteria B2b(ii)). Following the IUCN Red List Categories and Criteria (IUCN 2001), this species has been classified as Vulnerable (VU). As there is no possibilities of rescue from neighboring regions, hence there is no change to the initial assessment.

10. Nostolachma khasiana (Korth.) Deb \& Lahiri in Bull. Bot. Surv. India 17: 162. 1975. Lachnastoma khasiana Korth., Ned. Kruidk. Arch. 2(2): 202. 1851. Coffea khasiana (Korth.) Hook.f., Fl. Brit. India 3: 154. 1880; Kanjilal et al., FI. Assam 3: 74. 1939.

Shrub, up to $2.7 \mathrm{~m}$ high; young twigs pubescent. Leaves oblong or elliptic-lanceolate, $6.5-12 \times 2-3.8 \mathrm{~cm}$, caudateacuminate, sinuate, glabrous above; lateral nerves $6-8$ pairs; petiole short, $0.5-0.6 \mathrm{~cm}$ long, stout pilose. Stipules subulate from base, ca. $0.7 \mathrm{~cm}$ long. Flowers ca. $0.6 \mathrm{~cm}$ long, many in axillary dense much branched capitulate pubescent cymes. Calyx ca. $0.12 \mathrm{~cm}$ long; teeth 4, pilose, acute. Corolla green in bud ultimately orange; tube ca. $0.5 \mathrm{~cm}$ long; throat villous; lobes 4 , triangular, ca. $0.12 \mathrm{~cm}$ 
long. Stamens 4, fixed at throat of corolla tube; filaments short. Ovary 2-celled with one pendulous ovule in each surmounted by an annular fleshy disk; style short; stigma deeply bifid; lobes linear. Drupe globose, ca. $7 \times 5 \mathrm{~mm}$, crowned by persistent calyx teeth; seeds ventrally concave.

Flowering \& Fruiting: April-February.

Distribution: India [Assam (Cachar, GoalparaGuma R.F., Lakhimpur-Dullung R.F., Sibsagar), Manipur, Meghalaya, Mizoram, Tripura, Nagaland, Arunachal Pradesh]; Bhutan; Myanmar.

Specimens examined: 74121 (ASSAM), 23.ix.1978, Assam, Cachar, Mohankhal-Barunkhal, coll. R.B. Majumdar; 84268 (ASSAM), 19.xi.1983, Lailapur, R.B. Majumdar.

Global Red List Category: Not Evaluated.

Regional Red List Category and Criteria: The area of occupancy is calculated to be ca. $501 \mathrm{~km}^{2}$ (Criteria VU B2 $<2,000 \mathrm{~km}^{2}$ ), currently known to exist in 5-6 locations (Criteria VU B2a $\leq 10)$; the area of occupancy is rapidly decreasing due to slash-and-burn cultivation (Criteria VU B2b(ii)). According to IUCN Red List Categories and Criteria (IUCN 2001), it meets the criteria for Vulnerable (VU), but due to rescue possibilities from surrounding regions, it is downgraded by one step to Near Threatened (NT).

11. Ophiorrhiza hispida Hook.f., Fl. Brit. India 3: 83. 1880; Kanjilal et al., Fl. Assam 3: 42. 1939; Deb \& Mondal in Bull. Bot. Surv. India 39: 59. 1997.

Procumbent hispid herb, ca. $35 \mathrm{~cm}$ high. Leaves ovatelanceolate, $7.5-14.5 \times 3-4.5 \mathrm{~cm}$; lateral nerves $6-12$ on either side; petiole $0.5-1.8 \mathrm{~cm}$ long. Stipules oblonglanceolate, acuminate. Inflorescence terminal capitate cymes, 1-2 cm across, subglobose. Flowers greenishwhite or reddish, bracteate, epigynous, pedicellate, 5-6.5 $\mathrm{cm}$ long. Calyx obovoid; lobes 5 , ca. $1 \times 0.5 \mathrm{~mm}$, ovatelanceolate, acute. Corolla $0.4-0.5 \mathrm{~cm}$ long, shortly funnel shaped, hispid outside, villous at throat within; lobes 5, ca. $1.5 \times 0.8 \mathrm{~mm}$, ovate-oblong, spreading, acute. Stamens 5 , adnate to throat of corolla; anthers 1-1.25 mm long, linear-oblong; filaments ca. $1 \mathrm{~mm}$ long, glabrous. Ovary obovoid to subglobose; styles 1-1.5 mm long, glabrous; stigma 2-lobed. Capsule ovate-oblong, hispid, 2-locular; seeds brownish, 5-7 angular, glabrous.

Flowering \& Fruiting: August-December.

Distribution: India [Assam (Cachar, Digboi), Meghalaya]; China.

Specimens examined: 73429 (ASSAM), 19.xi.1978, Assam, Cachar, Lailapur, Aizwal Road, coll. R.B. Majumdar; 73057 (ASSAM), 08.xi.1978, Bhuban Hill, coll. R.B. Majumdar.

Global Red List Category: Endangered (Walter \& Gillett, 1998).

Regional Red List Category and Criteria: The Extent of occurrence is estimated to be ca. $417 \mathrm{~km}^{2}$ (Criteria EN B1 $\left.<5,000 \mathrm{~km}^{2}\right)$; area of occupancy estimated to be ca. $82.5 \mathrm{~km}^{2}$ (Criteria B2 $<500 \mathrm{~km}^{2}$ ), currently known to exist in three locations (Criteria EN B2a $\leq 5$ ); there is a continuous decline in area, extent and quality of habitat due to encroachment of forest land (Criteria EN B2b(iii)). Following the IUCN Red List Categories and Criteria (IUCN
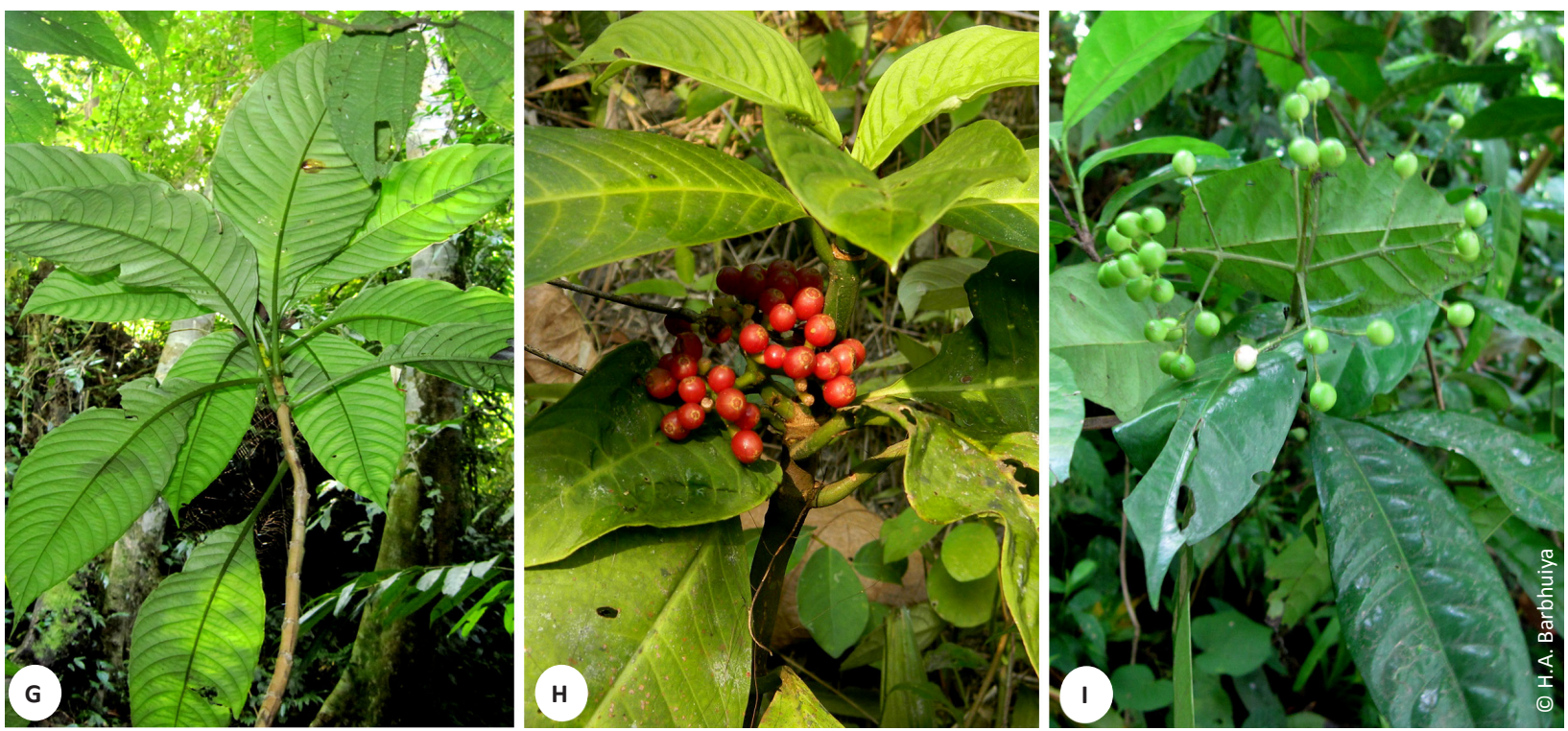

Image 2. G - Mycetia mukerjiana Deb \& R.M.Dutta.; H - Psychotria silhetensis Hook. f. subsp. silhetensis; I - Tarenna campaniflora (Hook.f.) N.P. Balakr. 
2001), this species has been classified as Endangered (EN). As there is no possibilities of rescue from neighboring regions, hence there is no change to the initial assessment.

12. Ophiorrhiza repens (Wall. ex G. Don) Bennet in Indian Forester 108: 302. 1982; Deb \& Mondal in Bull. Bot. Surv. India 39: 99. 1997. Ophiorrhiza calcarata Hook. f., FI. Brit. India 3: 84 1880. Mussaenda repens Wall. ex G. Don, Gen. Hist. 3: 491.1834.

Perennial herb. Stem slender, erect, $25-50 \mathrm{~cm}$ high. Leaves elliptic-lanceolate, 3-12 × 1.5-4 cm long, lurid green above, pale beneath, acuminate, glabrous above; petiole 1-1.5 cm long. Stipules lanceolate, entire or 2-fid. Cymes 8-20-flowered; branches short ascending; bracteoles much shorter than flowers, linear pubescent. Calyx 2-3 × 1-1.5 mm, pubescent; lobes 5, 1-1.25 × 0.5 $\mathrm{mm}$, ovate-lanceolate, acute, pubescent. Corolla white, 2-2.5 cm, tube narrow, puberulous or tomentose, throat hardly dilated; lobes 5 , erect, $0.75-1 \times 0.5 \mathrm{~mm}$, ovate, acute, much shorter than their recurved hispid dorsal spur, glabrous within. Stamens inserted at the middle of corolla; filaments $0.75-1.25 \mathrm{~mm}$ long; anthers ca. 2.5 $\mathrm{mm}$ long, linear-oblong. Ovary 1-1.5 × 0.75-1.25 mm, obovoid; style 10-22 mm long; stigma-lobed, ca. $1.5 \mathrm{~mm}$ long; lobes elliptic-lanceolate, glabrous. Capsule 2-3.5 $\times$ 2-8.5 mm, pubescent.

Flowering \& Fruiting: September-March.

Distribution: India [Assam (Cachar), Arunachal Pradesh, Meghalaya, Mizoram, West Bengal]; Bhutan; Myanmar.

Specimens examined: 73181 (ASSAM), 10.ix.1978, Assam, Cachar, Amraghat, coll. R.B. Majumdar; 914 (ASSAM), 03.vi.2012, Bhuban Hill, coll. H.A. Barbhuiya.

\section{Global Red List Category: Not Evaluated}

Regional Red List Category and Criteria: The area of occupancy is estimated to be ca. $503 \mathrm{~km}^{2}$ (Criteria VU $\mathrm{B} 2<2,000 \mathrm{~km}^{2}$ ), currently known to exist in six locations (Criteria VU $\mathrm{B} 2 \mathrm{~b} \leq 10)$; there is a continuous decline in area, extent and quality of habitat due to encroachment of forest land (Criteria VU B2b(iii)). According to IUCN Red List Categories and Criteria (IUCN 2001), it meets the criteria for Vulnerable (VU), but due to rescue possibilities from adjacent regions, it is downgraded by one step to Near Threatened (NT) regionally.

13. Psychotria silhetensis Hook. f., Fl. Brit. India 3: 174. 1880; Deb \& M. Gangop. in J. Econ. Taxon. Bot., Addit. Ser. 7: 144. 1989. Uragoga silhetensis (Hook. f.) Kuntze, Revis. Gen. PI. 2: 962.1891.

subsp. silhetensis (Image 2(H).

Shrub ca. $1 \mathrm{~m}$ high; branches glabrous. Leaves oblong- elliptic, 13-24 × 6-9 cm, acuminate or acute at apex, cuneate at base, glabrous; lateral nerves 13-20 on either side, subparallel; petiole 1-1.5 cm long, glabrous. Stipules ovate-oblong, $1-2.5 \times 0.5-1 \mathrm{~cm}$, acuminate at apex, glabrous. Inflorescence terminal or axillary cymes, 2-4 $\times$ $2-2.5 \mathrm{~cm}$, fruiting branches spreading, deflexed; peduncles 3-4 cm long, thick, glabrous; bracteoles minute, deltoid. Flowers greenish brown or white, pedicellate, 3-5 mm long, short tubular; pedicels $0.5-1 \mathrm{~mm}$ long, puberulous. Calyx 1.5-2 × 2-2.5 mm, cupular, puberulous above; lobes 5 , ovate-lanceolate, acute at apex. Corolla tube 1.5-3 mm long, glabrous above, densely velutinous at throat within; lobes 5, 1-2 mm long, ovate-lanceolate. Stamens 5, inserted; filaments $0.5-0.7 \mathrm{~mm}$ long; anthers lanceolate, 0.5-0.8 mm long. Ovary ca. $0.5 \mathrm{~mm}$; style $0.5-0.7 \mathrm{~mm}$ long; stigma 2-lobed. Fruits stalked, 8-13 × 5-6 mm, deep reddish when ripe.

Flowering \& Fruiting: June-January.

Distribution: India [Assam (Cachar, Hailakandi, Mikir Hills-Kaliani, Kamrup-Bamunigaon), Meghalaya, Mizoram, Arunachal Pradesh]; Bangladesh.

Specimen examined: 855 (ASSAM), 06.i.2012, Assam, Cachar, Inner Line Reserve Forest, Near Loharbond, coll. H.A. Barbhuiya

Global Red List Category: Not Evaluated

Regional Red List Category and Criteria: Area of occupancy estimated to be ca. $501 \mathrm{~km}^{2}$ (Criteria VU $\left.\mathrm{B} 2<2,000 \mathrm{~km}^{2}\right)$, currently known to exist in 6 locations (Criteria VU B2as10); the quality of habitat is rapidly decreasing due to slash-and-burn cultivation and encroachment of forest land (Criteria VU B2b(iii)). Following the IUCN Red List Categories and Criteria (IUCN 2001), this species has been classified as Vulnerable (VU). As there is no possibilities of rescue from neighboring regions, hence there is no change to the initial assessment.

14. Tarenna campaniflora (Hook.f.) N.P. Balakr. in Bull. Bot. Surv. India 22: 175. 1980. Webera campaniflora Hook.f., Fl. Brit. India 3: 106. 1880; Kanjilal et al., Fl. Assam 3: 53. 1939. Ixora campaniflora (Hook.f.) Kuntze, Revis. Gen. PI. 1: 278. 1891. Stylocoryna campaniflora (Hook.f.) K. Schum., Nat. Pflanzenfam. 4(4): 108. 1891. Image 2(I).

Shrub or small tree. Leaves elliptic or elliptic-lanceolate, $7.6-14 \times 2.5-5.8 \mathrm{~cm}$, entire, shortly acuminate, acute at base or occasionally rounded; glabrous above, pubescent or puberulous beneath; lateral nerves $12-15$ on either side; petiole $0.7-1.8 \mathrm{~cm}$ long. Stipules deciduous. Flower in terminal corymbose cymes; bracteoles two on each pedicel. Calyx hirsute; lobes 5, ovate-lanceolate, longer than the ovary. Corolla $1.5-2 \mathrm{~cm}$ long with a short villous 
tube, spreading into a large campanulate limb, 1.2-1.6 $\mathrm{cm}$ in dia.; lobes 5 , short ovate, acute, glabrous. Stamens 5 , fixed on the throat of the corolla; anthers long sessile. Ovary 2-celled, one ovule in each cell; style short, hairy; stigma long, fusiform. Fruit globose, 6-8 $\mathrm{mm}$ in diam.

Flowering \& Fruiting: August-December.

Distribution: India [Assam (Cachar, N.C. Hill-Haflong, Karbi Anglong-Dipu R.F., Bokajan, Sibsagar)]; Bangladesh; Myanmar.

Specimen examined: 4724 (ASSAM), 18.xi.1914, Assam, Cachar, Phulertal, coll. U.N. Kanjilal; 112233 (ASSAM), 17.viii.2013, Lower Jiri Reserve Forest, coll. H.A. Barbhuiya.

\section{Global Red List Category: Not Evaluated}

Regional Red List Category and Criteria: The Extent of occurrence is estimated to be ca. $101 \mathrm{~km}^{2}$ (Criteria EN B1<5,000 $\left.\mathrm{km}^{2}\right)$; Currently known from about three locations (Criteria ENB2a<5). The area of occupancy is estimated to be ca. $60 \mathrm{~km}^{2}$ (Criteria EN B2<500 km²); there is a continuous decline in area, extent and quality of habitat due to encroachment of forest land (Criteria EN B2b(iii)). Following IUCN Red List Categories and Criteria (IUCN 2001), it has been categorized as Endangered (EN). As there is no possibilities of rescue from neighboring regions, hence there is no change to the initial assessment.

\section{Conclusions}

The Rubiaceae members are ecologically sensitive and in combination with other factors such as restricted distribution, they are vulnerable to extinction (Davis et al. 2009). With the increase in human population, expansion of agricultural activities, over-exploitation of forests for firewood, shifting cultivation, extensive timbering, construction of roads and other developmental activities, the forest cover of the region is rapidly shrinking and as a result the threatened taxa of the region become more endangered. At present southern Assam has 116 tea gardens covering $8.14 \%$ of the total geographical area of the region. Tropical rainforests were massively destroyed for the establishment of tea gardens from 1858-1937. It is obvious that herbaceous plants discovered prior or during that period had nearly disappeared, particularly those which had a limited population or were too restricted in distribution.

The present study has allowed us to identify 14 threatened taxa, of which two are already extinct from the region while the remaining are to be conserved as top priority. Efforts should be made to protect and preserve the particular localities and habitats of these plants. The germplasm of all the surviving threatened taxa should be conserved in botanic gardens as ex situ conservation. It is suggested that these plants be multiplied through tissue culture techniques and reintroduce the propagated clones to their natural habitats.

\section{References}

Brandis, D. (1906). Indian Trees: An Account of Trees, Shrubs, Woody Climbers, Bamboos and Palms Indigenous or Commonly Cultivated in the British Indian Empire. Archibald Constable \& Co. Ltd, London, xxxiv+767pp; http://dx.doi.org/10.5962/bhl.title.50463

Champion, H.G. \& A.K. Seth (1968). A Revised Survey of the Forest Types of India. Manager of Publications, Govt. of India, New Delhi, xxvii+404pp.

Chowdhury, S. (2005). Assam's Flora, Present Status of Vascular Plants. Assam Science Technology and Environmental Council, Guwahati, 368pp.

Das, S.N. \& S.C. Roy (1984). A note on the occurrence of Chassalia staintonii (Hara) Deb \& Mondal (Rubiaceae) in India. Journal of Economic and Taxonomic Botany 5(2): 473-474.

Davis, A.P., R. Govaerts, D.M. Bridson, M. Ruhsam, J. Moat \& N.A. Brummitt (2009). A Global Assessment of Distribution, Diversity, Endemism, and Taxonomic Effort in the Rubiaceae. Annals of the Missouri Botanical Garden 96: 68-78.

Deb, D.B. (2001). Study of floristics and plant taxonomy. Phytotaxonomy 1: 5-17.

Deb, D.B. \& D.C. Mondal (1982). On the identity of Ophiorrhiza staintonii Hara (Rubiaceae). Journal of Japanese botany 57(5): 159-160.

Deb, D.B. \& B. Krishna (1982). Taxonomic studies of the genus Chassalia Comm. ex Poir, in India and adjoining regions. Bulletin of the Botanical Survey of India 24(1-4): 221-224.

Google Earth 6.1.0.5001 (2011). Available from http://www.google. com/earth

Hara, H. (1977). New or noteworthy flowering plants from Eastern Himalaya: 20. Journal of Japanese botany 52(12): 353-359.

Haviland, G.D. (1897). A Revision of the Tribe Naucleeæ (Nat. Ord. Rubiaceæ). The Journal of the Linnean Society, Botany 33(228): 1-94; http://dx.doi.org/10.1111/j.1095-8339.1897.tb00653.x

Hooker, J.D. (1880). The Flora of British India. Vol. 3. L. Reeve \& Co., London, 712pp; http://dx.doi.org/10.5962/bhl.title.678

IUCN (2001). IUCN Red List Categories \& Criteria: Version 3.1. IUCN Species Survival Commission. World Conservation Union, Gland, Switzerland and Cambridge, United Kingdom, ii+30pp.

IUCN (2003). Guidelines for Application of IUCN Red List Criteria at Regional Levels: Version 3.0. IUCN Species Survival Commission. IUCN, Gland, Switzerland and Cambridge, United Kingdom, ii+26 pp.

IUCN (2012). IUCN Red List of Threatened Species. Version 2012.1. <www.iucnredlist.org>. Viewed on 28 August 2012.

Kanjilal, U.N., A. Das, P.C. Kanjilal, \& R.N. De (1939). Flora of Assam. Vol. 3. Government of Assam, Shillong, $x+578 p p$.

Kar, S.K. \& G. Panigrahi (1963). The Rubiaceae in Assam and North East Frontier Agency. Bulletin of the Botanical Survey of India 5(3\&4): 227237.

Sgrillo, R. (2012). GE-Path: Make Path for Google Earth v 1.4.6. Ricardo Sgrillo, Cocoa Research Center (CEPLAC/CEPEC), Ilheus, Bahia, Brasil. <http://www.sgrillo.net/googleearth/gepath. htm> Downloaded on 1 January 2012.

Sambamurthy, A.V.S.S. (2005). Taxonomy of Angiosperms. I. K. International Pvt. Ltd. New Delhi, v+908 pp.

Walter, K.S. \& H.J. Gillett (eds.) (1998). 1997 IUCN Red List of Threatened Plants. Compiled by the World Conservation Monitoring Centre. IUCN-The World Conservation Union, Gland, Switzerland, and Cambridge, United Kingdom, Ixiv+862pp; http://dx.doi.org/10.5962/ bhl.title. 44833

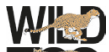

